\title{
El cariotipo de Chaetanthera linearis Poepp. (Asteraceae)
}

\section{The karyotype of Chaethanthera linearis Poepp. (Asteraceae)}

\author{
Carlos M. Baeza ${ }^{1}$, Eduardo Ruiz ${ }^{1}$, Patricio Novoa $^{2}$ \& María A. Negritto ${ }^{1}$ \\ ${ }^{1}$ Departamento de Botánica, Universidad de Concepción, Casilla 160-C, Concepción, Chile. \\ 2Jardin Botánico Nacional, camino Olivar 305, El Salto, Viña del Mar, Chile \\ cbaeza@udec.cl
}

\begin{abstract}
The karyotype of Chaetanthera linearis var. linearis from Chile was examined. The species has $2 \mathrm{n}=2 \mathrm{x}=22$ chromosomes, with $9 \mathrm{~m}+1 \mathrm{st}+1$ st-sat chromosomes. The reported karyotype is symmetric (AsK $\%=58.0, \mathrm{TF} \%=42.0$ and Syi $\%$ $=72.4$ ). The karyotype of this species was compared with Ch. microphylla var. microphylla a very similar species in morphology and distribution to Ch. linearis var. linearis, but with clearly differentiated karyotype.
\end{abstract}

Chaetanthera Ruiz et Pav. es un género sudamericano con alrededor de 42 especies, de las cuales 36 crecen en Chile (Cabrera 1937, Torres et al. 2007, Baeza et al. 2009). Cabrera (1937) reconoce dentro de C. linearis tres variedades, separándolas por la longitud de las lígulas y por la coloración de las flores marginales. Las plantas con lígulas más largas que el involucro y capítulos con flores marginales de color amarillo corresponden a la variedad típica; las de capítulos con flores blancas a la variedad albiflora Phil. y las plantas con lígulas más cortas que el involucro corresponden a la variedad taltalensis I.M.Johnst. En el mismo trabajo, Cabrera indica que $C$. linearis es una especie muy relacionada con C. microphylla (Cass.) Hook et Arn., pero con diferencias morfológicas suficientes para considerarlas especies distintas. Sobre la base de la coloración de las flores marginales, Cabrera (1937) reconoce dos variedades en C. microphylla, la variedad típica con flores rojizas y la variedad albiflora con flores blancas.

Existen antecedentes previos acerca del número cromosómico de C. linearis var. linearis. Grau (1987), señala $2 n=22$ para esta variedad de material proveniente de las localidades de Farellones, en la Región Metropolitana de Santiago y de la Cuesta La Dormida, en la provincia de Quillota. Además, el mismo autor señala $2 \mathrm{n}=24$ para $C h$. linearis var. albiflora, a partir de material recolectado en el Parque Nacional Fray Jorge, en la provincia de Limarí. Hershkovitz et al. (2006), basándose fundamentalmente en el análisis secuencial de la región ITS del ADN ribosomal nuclear, realizan un estudio evolutivo en Chaetanthera y observaron que $C$. microphylla y $C$. linearis siempre aparecen como especies estrechamente relacionadas.
Chaetanthera linearis var. linearis es una variedad endémica, anual, distribuida en las provincias centrales de Chile, preferentemente en enclaves secos y arenosos. Es una plantita baja, con flores liguladas o marginales amarillas.

Se estudió citológicamente una población de Chaetanthera linearis var. linearis recolectada en Chile, V Región, Provincia de Valparaíso, Dunas de Concón, Santuario, $103 \mathrm{~m}\left(32^{\circ} 56^{\prime} / 71^{\circ} 32^{\prime}\right)$, 11-XII-2007. C. Baeza \& P. Novoa 4271 a. El material de referencia está depositado en el Herbario de la Universidad de Concepción (CONC).

Se analizaron 10 placas metafásicas (procedentes de 10 individuos) y se midieron los cromosomas siguiendo el método propuesto por Baeza et al. (2006). Para la población analizada se confeccionó el cariotipo y el idiograma haploide, se determinó el índice de asimetría (AsK \%) definido por Arano \& Saito (1980), el TF\% = índice de asimetría de Huziwara (1962), el Syi = índice de asimetría de Venora et al. (2002), el cociente entre el par de cromosomas más largo y el más corto $(\mathrm{R})$ y la longitud total del genoma (LTC, expresada en $\mu \mathrm{m}$ ). Los cromosomas fueron clasificados de acuerdo a Levan et al. (1964).

Chaetanthera linearis var. linearis presenta $2 \mathrm{n}=2 \mathrm{x}$ $=22$ cromosomas (Fig. 1A), lo que confirma el número cromosómico documentado por Grau (1987), con un cariotipo claramente simétrico y un complemento cromosómico haploide de 9 pares de cromosomas metacéntricos y dos pares subtelocéntricos, el par 11 con un satélite en el brazo corto (Tabla I, Fig. 1 B-C). El índice de asimetría del cariotipo (AsK \%) fue de 58,0, el TF\% 42 , el Syi 72,4, la longitud total del genoma (LTC) fue de $81,8 \pm 8,50 \mu \mathrm{m}$ y $\mathrm{R}$ fue 2,08 (Tabla I). La longitud 
de los cromosomas fluctúa entre 5-2,4 $\mu \mathrm{m}$ (Tabla II). La Tabla I resume los datos citológicos de $C$. linearis var. linearis y C. microphylla var. microphylla (Baeza \& Schrader 2005), dos taxones de ciclo anual muy próximos morfológicamente. Todas las características cariológicas analizadas permiten concluir que se trata de dos entidades citológicas claramente definidas y diferenciadas por su cariotipo, lo que constituye un argumento de apoyo al criterio taxonómico de Cabrera (1937), quien señala que a pesar de ser taxones muy parecidos, muestran características morfológicas suficientes como para considerarlas especies distintas. Hasta ahora existen tres recuentos cromosómicos en Ch. linearis var. linearis y todos indican $2 \mathrm{n}=22$ cromosomas. La única excepción es Ch. linearis var. albiflora que presenta $2 \mathrm{n}=24$ (Grau 1987). Habría que prestar más atención a las variedades morfológicas reconocidas por Cabrera (1937), algunas de ellas estudiadas cariológicamente con detalle permitirían modificar su estatus taxonómico.
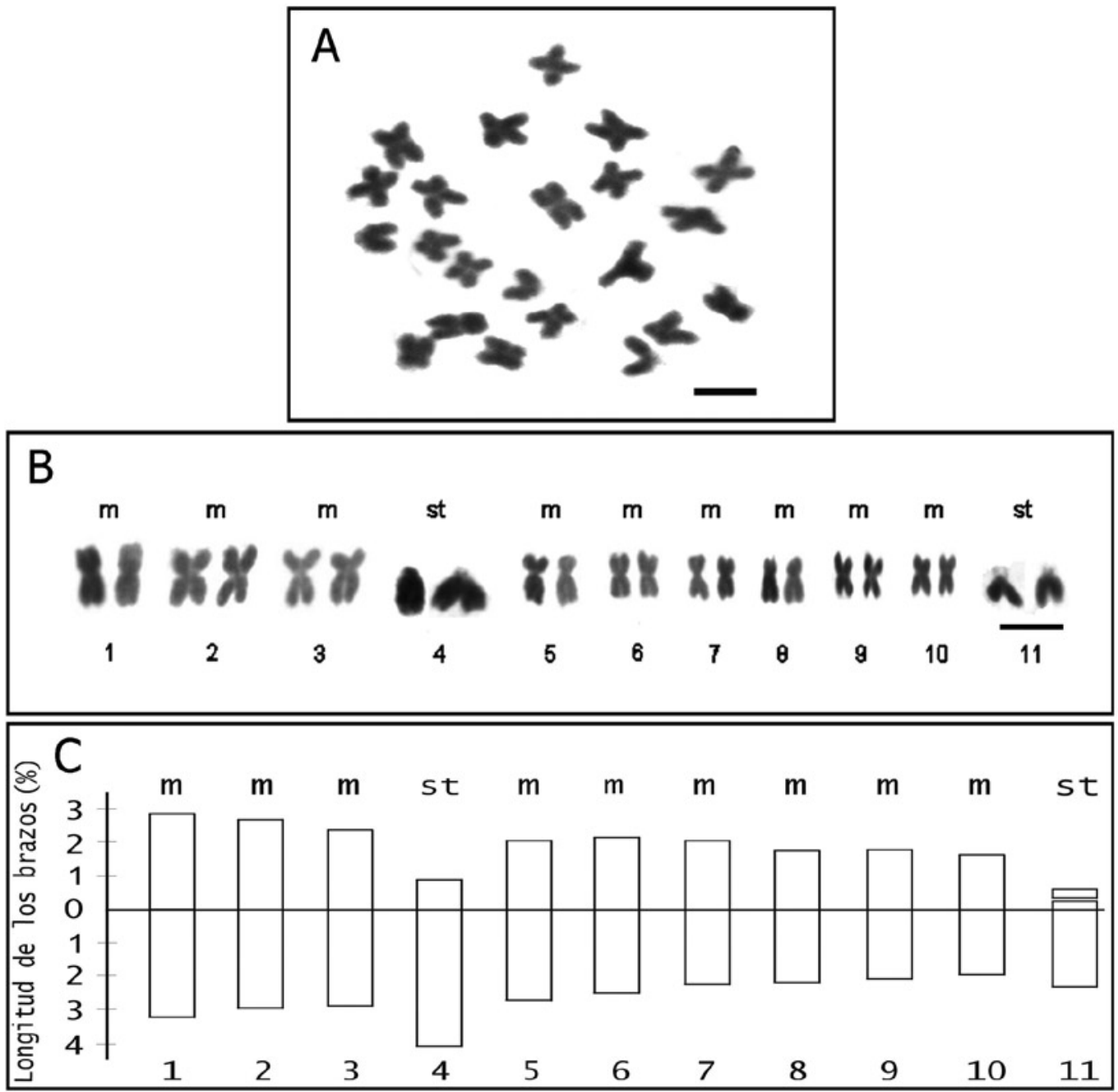

Figura 1. Chaetanthera linearis var. linearis. A. Placa metafásica $(2 \mathrm{n}=22)$. B. Cariotipo. C. Idiograma del complemento haploide $($ los cromosomas se han ordenado de acuerdo a su tamaño decreciente). Las escalas corresponden a $5 \mu \mathrm{m}$.

FIgURE 1. A. Metaphasic chromosomes of Chaetanthera linearis var. linearis $(2 \mathrm{n}=22)$. B. Karyotype. C. Ideogram of the haploid chromosomes complement (the chromosomes have been ordered according to decreasing size). Scales $=5 \mu \mathrm{m}$. 
Cariotipo de Chaetanthera linearis: BAEZA, C.M. ET AL.

TABLA I. Características del cariotipo de Chaetanthera linearis var. linearis y C. microphylla var. microphylla. LTC: longitud total de los cromosomas $(\mu \mathrm{m}) ;$ AsK \% = índice de asimetría de Arano \& Saito (1980), TF\% = índice de asimetría de Huziwara (1962), Syi = índice de asimetría de Venora et al. (2002); R: cociente entre el par de cromosomas más largo y el más corto.

TABLE I. Comparison of karyotype characteristics of Chaetanthera linearis var. linearis and C. microphylla var. microphylla. LTC: total chromosome length $(\mu \mathrm{m})$; AsK \% = Asymmetry index of Arano \& Saito (1980), TF\% = Asymmetry index of Huziwara (1962), Syi $=$ Asymmetry index of Venora et al. (2002); R: ratio of the longest pair/shortest pair.

\begin{tabular}{lcc}
\hline CARActerísticas del CARIOTIPO & C. linearis & C. microphylla \\
\hline 2n & 22 & 24 \\
Fórmula cariotípica & $9 \mathrm{~m}+1 \mathrm{st}+1$ st-sat & $8 \mathrm{~m}+4 \mathrm{st}$ \\
LTC $(\mu \mathrm{m})$ & $81,8 \pm 6,2$ & $93,5 \pm 9,7$ \\
$\mathrm{R}$ & 2,08 & 2,15 \\
AsK \% & 58,0 & 62,3 \\
TF\% & 42,0 & 37,75 \\
Syi & 72,4 & 60,6 \\
\hline
\end{tabular}

TABla II. Mediciones cromosómicas de Chaetanthera linearis var. linearis (C. Baeza \& P. Novoa 4271 a). Se detallan las longitudes promedio como porcentaje de la longitud del genoma haploide de 10 metafases.

TABLE II. Chromosomal measurements of Chaetanthera linearis var. linearis (C. Baeza \& P. Novoa 4271 a), calculated in percent of the mean haploid genome length of 10 metaphases.

\begin{tabular}{|c|c|c|c|c|c|}
\hline $\begin{array}{c}\text { PAR } \\
\text { CROMOSÓMICO }\end{array}$ & $\begin{array}{l}\text { BRAZO LARGO } \\
(\%) \pm \text { D.S. }\end{array}$ & $\begin{array}{c}\text { BRAZO CORTO } \\
(\%) \pm \text { D.S. }\end{array}$ & $\begin{array}{c}\text { LARGO } \\
\text { RELATIVO (\%) }\end{array}$ & $\begin{array}{l}\text { LARGO TOTAL } \\
\qquad(\mu \mathrm{m})\end{array}$ & TIPO DE CROMOSOMA \\
\hline 1 & $3,21 \pm 0,12$ & $2,86 \pm 0,12$ & 6,07 & 5,0 & $\mathrm{~m}$ \\
\hline 2 & $2,94 \pm 0,10$ & $2,69 \pm 0,10$ & 5,63 & 4,7 & $\mathrm{~m}$ \\
\hline 3 & $2,86 \pm 0,08$ & $2,39 \pm 0,10$ & 5,25 & 4,3 & $\mathrm{~m}$ \\
\hline 4 & $4,07 \pm 0,10$ & $0,90 \pm 0,10$ & 4,97 & 4,1 & st \\
\hline 5 & $2,70 \pm 0,14$ & $2,06 \pm 0,12$ & 4,76 & 3,9 & $\mathrm{~m}$ \\
\hline 6 & $2,48 \pm 0,10$ & $2,17 \pm 0,12$ & 4,65 & 3,8 & $\mathrm{~m}$ \\
\hline 7 & $2,22 \pm 0,12$ & $2,06 \pm 0,10$ & 4,28 & 3,5 & $\mathrm{~m}$ \\
\hline 8 & $2,17 \pm 0,10$ & $1,76 \pm 0,10$ & 3,93 & 3,2 & $\mathrm{~m}$ \\
\hline 9 & $2,05 \pm 0,10$ & $1,79 \pm 0,10$ & 3,84 & 3,1 & $\mathrm{~m}$ \\
\hline 10 & $1,93 \pm 0,12$ & $1,64 \pm 0,12$ & 3,57 & 2,9 & $\mathrm{~m}$ \\
\hline 11 & $2,29 \pm 0,10$ & $0,62 \pm 0,10$ & 2,91 & 2,4 & st-sat \\
\hline
\end{tabular}




\section{AGRADECIMIENTOS}

Se agradece el apoyo de Fondecyt No 1070520 y al Departamento de Botánica de la Universidad de Concepción por las facilidades otorgadas.

\section{BIBLIOGRAFÍA}

Arano, H. \& H. Saito. 1980. Cytological studies in family Umbelliferae 5. Karyotypes of seven species in subtribe Seselinae. La Kromosomo 2: 471-480.

BAEZA, C. \& O. Schrader. 2005. Análisis del cariotipo y detección de los genes 5S y 18S/25S rDNA en Chaetanthera microphylla (Cass.) H. et A. (Asteraceae). Gayana Botánica 62(1): 49-51.

Baeza, M., O. Schrader, E. Ruiz \& M. Negritto. 2006. Análisis comparativo del cariotipo en poblaciones de Alstroemeria ligtu subsp. ligtu y A. ligtu subsp. simsii (Alstroemeriaceae) de Chile. Darwiniana 44(2): 313-318.

BAeZA, M., E. RuIZ \& M. NegritTo. 2009. Importancia del cariotipo en la taxonomía y evolución del género Chaetanthera (Asteraceae): evidencias preliminares para especies que crecen en Chile. Gayana Botánica 66(1): 51-58.
Cabrera, A. 1937. Revisión del género Chaetanthera (Compositae). Revista del Museo de La Plata Sección Botánica 1: 87-215.

GraU, J. 1987. Chromosomenzahlen chilenischer Mutisieen (Compositae). Botanische Jahrbücher 108: 229-237.

Hershkovitz, M., K. Arroyo, C. Bell \& L. Hinojosa. 2006. Phylogeny of Chaetanthera (Asteraceae: Mutisieae) reveals both ancient and recent origins of the high elevation lineages. Molecular Phylogenetics and Evolution 41: 594-605.

Huziwara, Y. 1962. Karyotype analysis in some genera of Compositae. VIII. Further studies on the chromosomes of Aster. American Journal of Botany 49: 116-119.

Levan, A, K. Fredga \& A. Sandberg. 1964. Nomenclature for centromeric position on chromosomes. Hereditas 52: 201-220.

Torres, C., L. Cavieres, C. Muñoz \& M. Arroyo 2007. Consecuencias de las variaciones microclimáticas sobre la visita de insectos polinizadores en dos especies de Chaetanthera (Asteraceae) en los Andes de Chile central. Revista Chilena de Historia Natural 80: 455-468.

Venora, G., S. Blangiforti, M. Ruffini Castiglioni, D. Pignone, F. Losavio \& R. CREMONINI. 2002. Chromatin organisation and computer arded karyotyping of Triticum durum Desf. cv Timilia. Caryologia 55: 91-98. 